
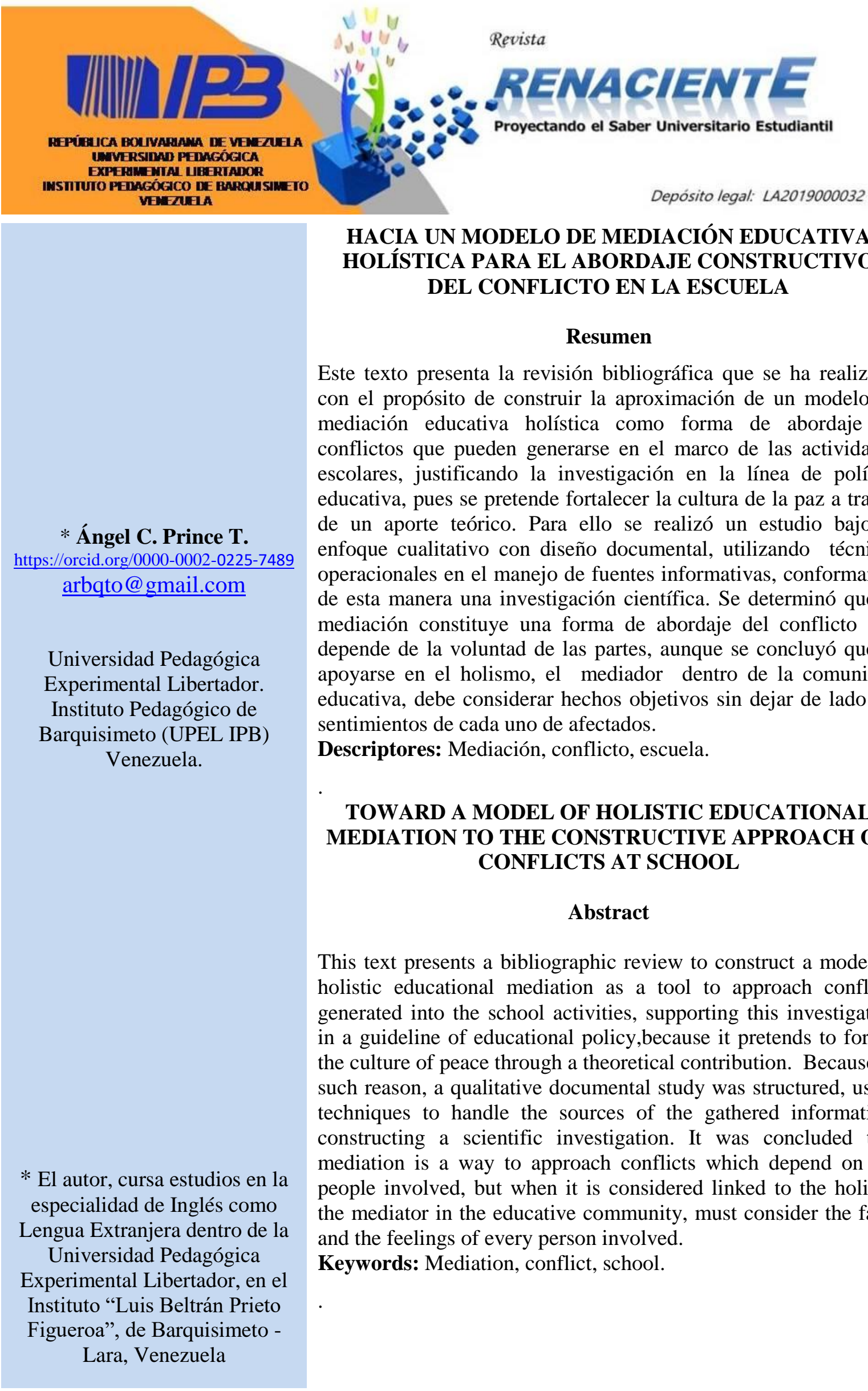

\title{
HACIA UN MODELO DE MEDIACIÓN EDUCATIVA HOLÍSTICA PARA EL ABORDAJE CONSTRUCTIVO DEL CONFLICTO EN LA ESCUELA
}

\section{Resumen}

Este texto presenta la revisión bibliográfica que se ha realizado con el propósito de construir la aproximación de un modelo de mediación educativa holística como forma de abordaje de conflictos que pueden generarse en el marco de las actividades escolares, justificando la investigación en la línea de política educativa, pues se pretende fortalecer la cultura de la paz a través de un aporte teórico. Para ello se realizó un estudio bajo el enfoque cualitativo con diseño documental, utilizando técnicas operacionales en el manejo de fuentes informativas, conformando de esta manera una investigación científica. Se determinó que la mediación constituye una forma de abordaje del conflicto que depende de la voluntad de las partes, aunque se concluyó que al apoyarse en el holismo, el mediador dentro de la comunidad educativa, debe considerar hechos objetivos sin dejar de lado los sentimientos de cada uno de afectados.

Descriptores: Mediación, conflicto, escuela.

\section{TOWARD A MODEL OF HOLISTIC EDUCATIONAL MEDIATION TO THE CONSTRUCTIVE APPROACH OF CONFLICTS AT SCHOOL}

\begin{abstract}
This text presents a bibliographic review to construct a model of holistic educational mediation as a tool to approach conflicts generated into the school activities, supporting this investigation in a guideline of educational policy,because it pretends to fortify the culture of peace through a theoretical contribution. Because of such reason, a qualitative documental study was structured, using techniques to handle the sources of the gathered information, constructing a scientific investigation. It was concluded that mediation is a way to approach conflicts which depend on the people involved, but when it is considered linked to the holism, the mediator in the educative community, must consider the facts and the feelings of every person involved.
\end{abstract}

Keywords: Mediation, conflict, school. 


\section{Introducción}

En el marco de las actividades que se desarrollan día a día dentro de las instituciones educativas que acogen en su seno a niños, adolescentes y adultos, se presenta el acaecimiento de diferentes conflictos en los cuales repetidamente se manifiesta la violencia física y/o psicológica. La aseveración previamente realizada es de evidente materialización, puesto que sólo basta ser partícipe como observador o como agente activo en los centros de educación inicial, las escuelas, los liceos y las universidades, para palpar cómo en el mismo momento en el que una persona acosa, insulta o incluso arremete físicamente contra otra, se comprueba lo ya mencionado. De hecho con respecto a este punto se indica que:

Según el plan internacional de la Unesco, alrededor de 246 millones de niños y adolescentes sufren algún tipo de violencia escolar. Conocer sus diferentes formas de manifestarse es fundamental para abordar este problema tanto dentro como fuera de las aulas (Universidad de la Rioja, 2020 p.1).

Por lo anterior, El maestro en sus distintos roles como académico, orientador, amigo, confidente, entre otros, podría abordar constructivamente estas situaciones conflictivas y para ello requiere desarrollar competencias adecuadas, tanto teóricas como prácticas. Sin embargo cabría plantearse si realmente los docentes conocen el papel crucial que juegan en este entorno y si cuentan con las herramientas necesarias para fomentar un ambiente académico de cordialidad, en el cual puedan llevarse a cabo sin elementos conflictivos las actividades que normalmente se generan en las instituciones educativas.

Cuando se hace referencia a la escuela, se manifiesta la visualización de un recinto académico en el cual se facilitan conocimientos dentro de un ambiente de socialización, donde están presentes diversas ideologías, culturas, etnias, religiones, entre otros elementos. De hecho, con respecto a las relaciones dentro del espacio en las instituciones de educación, se sostiene lo siguiente:

La escuela que cumple una función socializadora por medio de las relaciones que se vivencian en cada jornada académica, por las actividades escolares que diariamente involucran a los estudiantes en procesos de socialización, los diálogos y los encuentros en los espacios de descanso y esparcimiento que son permitidos en abordaje constructivo del conflicto en la escuela .(pp 110-135) 
el ámbito escolar en donde se comparten reflexiones, recuerdos, emociones, gustos y situaciones que llevan a establecer acuerdos y aceptar las diferencias con respeto y tolerancia, actividades que deben estar bajo la supervisión responsable y comprometida de adultos que hacen parte de la comunidad educativa (Bolaños, 2019, p.1).

Ahora bien, teniendo en cuenta el marco integrador antes indicado, conviene apuntar que en realidad no siempre el acto pedagógico se desenvuelve dentro de estas pautas un tanto utópicas y de hecho, el auge de la violencia mundial se hace latente en las instituciones educativas y por ello se ejemplifica este hecho al sostener que “...la mitad de los estudiantes de entre 13 y 15 años de todo el mundo -alrededor de 150 millonesdeclaran haber experimentado violencia entre pares en las escuelas y en sus inmediaciones" (Unicef, 2018, p.1). De la misma manera, muy conocido es el hecho de que los niños y adolescentes en las aulas, experimentan el fenómeno de la violencia a pesar de que estos espacios han sido diseñados con el propósito firme de desarrollar las relaciones interpersonales armónicamente, mientras que por otra parte obtienen herramientas para solucionar los conflictos en los cuales se ven envueltos.

Es por lo ya apuntado, que puede afirmarse que la incidencia de los antivalores como el egoísmo, la intolerancia, la falta de respeto por la propia persona o la asimilación de la violencia como forma de sobresalir o resolver disputas, ha sido determinante para potenciar a las agresiones en sus diferentes vertientes en las actividades realizadas dentro de los recintos destinados a la formación de los ciudadanos del mañana (ya que no sólo asestando un golpe o atacando con un arma, como comúnmente se cree, se hace presente esta suerte de "terror" en los centros de enseñanza). Por esto resulta imperativo investigar los aspectos planteados para identificar soluciones dirigidas a prevenir y contener los focos de violencia escolar, a manera de evitar los trastornos dentro de la comunidad educativa que pudieran suscitarse como consecuencia trascendente de la implicación de sujetos que activa o pasivamente se vean inmersos en tal fenómeno. De hecho, resulta pertinente expresar que:

Hay que destacar la necesidad de que se contrarresten estos problemas, también en beneficio de los alumnos agresores quienes, como señalábamos antes, están más 
expuestos que otros a seguir por caminos antisociales. Por tanto, es fundamental que intentemos frenar sus actividades y las dirijamos por canales socialmente más aceptables (Olweus, 1998, p. 69).

Precisamente, para limitar la vorágine de acciones negativas que representan los conflictos, se presentan los medios alternos (o adecuados, como también son llamados en la actualidad) de resolución de conflictos, como respuesta para confrontar las problemáticas desde una óptica constructiva, que permita legitimar a las partes involucradas y que aporte remedios para satisfacer las necesidades de todas aquellas personas que de uno u otro modo, necesitan de una voz que haga eco de sus requerimientos sin necesidad de llegar al litigio o a la agresión. Así, dentro de estas formas avanzadas de resolución de conflictos se encuentra la figura de la negociación asistida, conocida como mediación en la que se persigue la obtención de soluciones que representen ganancia para los involucrados en determinada contraposición de intereses.

Una vez esbozados los aspectos previos de este escrito, debe dejarse claro que como una estrategia para evitar, contener o solucionar la generación de violencia, se presenta la implementación de la mediación en su carácter de medio alterno para abordar conflictos, como una disciplina que podría conducir a acuerdos entre las partes involucradas, con intervención de una tercera persona neutral que impulsa la búsqueda de soluciones que surjan de los propios interesados en solventar la situación problemática, todo ello dentro de un enfoque ganar-ganar en el que cada uno obtenga un beneficio palpable que responda a sus necesidades. Es así como incluir la figura en cuestión dentro del concepto de pedagogía de la paz concebido como "un modelo de capacitación para generar cambios de percepción, de actitudes y de conducta" (Cuenca, 2006, p. 15), puede hacerse referencia a una variante denominada mediación educativa.

La mediación educativa se desenvuelve en el espectro de la educación para la paz, con la cual "se trata de rechazar la guerra, sí, pero también de educar en la tolerancia, la solidaridad, la justicia, la libertad, el diálogo, el cuidado del medio ambiente, la cooperación...” (Molina, 2020, p. 1) y serían estos sus pilares conformantes, que del mismo modo son fines supremos de las alternativas para resolver colisiones de necesidades. También cabe señalar que este tipo formación es una "tentativa de responder a los 
problemas de conflicto y de violencia en escalas que se extienden desde lo global y lo nacional a lo local y personal" (Hicks, 1999, p. 23). Es por ello que al hacer referencia a este modo de solucionar conflictos, no se trata más que de trasladar los parámetros de la mediación convencional que desde sus inicios tan efectiva ha resultado para solucionar las disputas, además de las manifestaciones individuales y colectivas de agresividad.

Todo lo apuntado conllevaría a estructurar, a través de estas líneas, un modelo de Mediación Educativa Holística susceptible de ser implementada en los recintos de estudio como iniciativa para prevenir, resolver o contener los hechos violentos potencialmente palpables en los mismos desde diversas perspectivas. El carácter holístico de la figura en cuestión, se adhiere a ella porque la intervención del tercero objetivo para solventar problemáticas con iniciativa en las propuestas de las partes afectadas, involucra no sólo a técnicas, estudios y destrezas comunicacionales, sino que también implica la toma en cuenta de sentimientos, valores, actitudes, antecedentes, trasfondos motivacionales de los involucrados y características psicológicas, es decir, representa un medio avanzado de abordaje del conflicto que se implementa como un proceso integral. No sólo se constriñe a la puesta en práctica de pericias, sino que debe obedecer a la búsqueda de acuerdos satisfactorios tomando en cuenta un abanico de supuestos que son muy sui géneris para cada caso.

Por lo antes referido, se presenta la necesidad de explicar que la voz griega holos significa "todo" y denota organización, lo que está entero, integrado y que no carece de factores para ser completado. Cuando se refiere al holismo, simplemente se hace alusión a una propensión a comprender los sucesos así como los asuntos desde una perspectiva que considere la interrelación de la totalidad de elementos que interactúan entre sí para conformarlo. De allí que Weil (1996) exponga que desde la perspectiva holística, la totalidad y a su vez la individualidad de sinergias se encuentran íntimamente vinculadas con interacciones persistentes, que pueden dar pie a la configuración de nuevos fenómenos o situaciones. Esta es la razón por la cual el modelo sugerido en esta investigación, ha sido denominado como mediación educativa holística.

Así, cabría realizarse las siguientes interrogantes: ¿resulta útil el diseño de un modelo de mediación desde el punto de vista holístico para lograr el abordaje constructivo 
del conflicto en la escuela?; ¿cuáles son las necesidades que impulsan la propuesta de un modelo de mediación holística? ; y finalmente, ¿qué papel juega la mediación como medio avanzado para abordar los conflictos? Por lo cual pueden entonces señalarse los propósitos de esta investigación. De esta manera el propósito general de este artículo es estudiar el acercamiento a un modelo de mediación educativa holística como forma de abordaje constructivo del conflicto en la escuela, mientras que específicamente se pretende: 1. Discutir la importancia de la mediación como medio adecuado para dirimir controversias; 2. Establecer las implicaciones de los conflictos y 3. Analizar a la mediación desde una perspectiva holística como medio alterno para prevenir, contener o resolver conflictos educativos.

\section{Argumentación teórica}

Una vez recabada la información pertinente sobre los conflictos y la mediación, es menester que la misma sea organizada de manera que se comprenda el producto de la agrupación de los documentos estudiados. De esta manera es como a continuación, se procede aquí a presentar el resultado de la compilación bibliográfica realizada a través de diferentes apartados que sustentan las bases relacionadas con el medio alterno de resolución de conflictos objeto de este trabajo.

\section{Conflictos y necesidades}

El conflicto, no es más que el fenómeno que se manifiesta al generarse una situación de intereses insatisfechos entre partes que toman una postura contrapuesta en la cual se manifiesta la diferencia de los individuos relativa a emociones, relaciones, percepciones, acepciones, valores, requerimientos, culturas y factores sociales. En este orden de ideas se expone que el conflicto se constituye como "un proceso dinámico en el cual los eventos y las comprensiones constantemente se reestructuran y reinterpretan el pasado, el presente y el futuro" (Cuenca, 2006, p. 23), por lo que este sistema puede tornarse en cuanto a su naturaleza en un producto constructivo o destructivo, dependiendo del abordaje al cual se sujete y a la evolución o involución que se manifieste en el tránsito de la búsqueda de reparos que se ideen para solventarlo, contenerlo o prevenirlo, y así en cuanto a su tipología puede clasificarse como:

115 Ángel Carmelo Prince Torres: Hacia un modelo de mediación educativa holística para el abordaje constructivo del conflicto en la escuela .(pp 110-135) 
a) Latente: Cuando se cultiva durante la interacción entre personas aunque no aflore de forma palpable.

b) Real: $\mathrm{Si}$ es percibido a través de los sentidos de manera evidente por los involucrados en la relación discordante.

c) Falso: Que se traduce en una incorrecta interpretación de la realidad por los implicados en la relación.

d) Contingente: El cual está circunscrito a factores en la relación del género humano, en métodos y procedimientos o en la funcionalidad de la dinámica generada, que afecta al conflicto propiamente dicho.

e) Constructivo: Si representa una oportunidad para el cambio, el aprendizaje y el crecimiento personal, aparte de hacer tangible la obtención de beneficios para todos los implicados en la contraposición de intereses.

f) Destructivo: Si se torna en una forma de exclusión o su producto resulta lesivo de los intereses de los implicados, pudiendo ramificarse según desemboque en una materialización del enfoque en el cual todos pierden o en el cual alguien gana, pero la otra persona pierde.

g) Simbólico: Que se encuentra fundado en el fuero imaginario de la sociedad o en una individualidad.

h) Desplazado: Es aquel en el cual se constituye la disputa entre agentes que no han sido responsables por la generación del conflicto o que no cuentan con las destrezas para buscar una solución satisfactoria, así como puede circunscribirse también a la oposición por motivos errados.

Así, se consolida la postura que desde el principio se ha mantenido a lo largo de esta investigación tendente a la formulación de un modelo de mediación educativa holística: si existe una necesidad insatisfecha, sencillamente se genera un conflicto. De hecho, "diversos estudios concluyen que son las conductas violentas menos graves, como la agresión verbal, las que se dan con mayor frecuencia" (Universidad Internacional de Valencia, 2018, p.1) y de este modo, cuando el conflicto como desacuerdo entre las partes se decanta en violencia, puede llegar al extremo de la destrucción de todo aquello que

116 Ángel Carmelo Prince Torres: Hacia un modelo de mediación educativa holística para el abordaje constructivo del conflicto en la escuela .(pp 110-135) 
dentro de la sociedad se ha tratado de construir, siendo aceptado para el enriquecimiento de la cultura y la convivencia.

Con respecto al tema, expone Galtung (1998), que la violencia puede clasificarse de la siguiente manera:

a) Violencia directa, cuando es ejecutada por una persona de forma intencional y quien resulta como víctima, es sujeto de perjuicios psicológicos y/o físicos, manifestándose esencialmente en relaciones asimétricas, es decir, aquellas en las cuales no hay paridad de condiciones entre las partes involucradas.

b) Violencia estructural, que se presenta al no existir un agente concreto que ejecute la acción, sino que puede obedecer a causas internas (cuando es generada desde el seno de la personalidad) o externas (si proviene del conglomerado social).

c) Violencia cultural, si es producto de elementos culturales que permiten a la religión, la esfera jurídica, las manifestaciones artísticas, los actos científicos o las ideas, adoptar acciones que vulneran la vida.

Conociendo todas estas nociones, debe resaltarse que reviste una gran importancia para la conformación de la personalidad del ser humano, ya que la adolescencia y la niñez son etapas que pueden marcar o no a un individuo, y por eso es de suma importancia que las problemáticas que con respecto a ellos se generen se aborden de forma idónea, siendo esta una perfecta oportunidad para crear y consolidar en ellos una conciencia pacificadora en la cual se considere su respeto propio y por los demás. Debe de esta forma asimilarse que los planteles educativos y sus actores juegan un rol determinante para formar a las personas, por lo que aquí es cuando entra en juego la mediación como una forma de inculcar la cultura de paz en el ambiente de los miembros de la comunidad educativa.

\section{La Mediación: Noción y alcance}

Con respecto a los medios alternos de solución de conflictos, se estima que "tienen como finalidad propiciar la solución de controversias que surjan entre los miembros de la sociedad con motivo de denuncias o querellas" (Couttolenc, 2020, p.1) mientras que además se sostiene que son no onerosos y flexibles. Dentro de ellos se incluye a la mediación, que“es el proceso de deliberación voluntario que es estructurado y facilitado por

117 Ángel Carmelo Prince Torres: Hacia un modelo de mediación educativa holística para el abordaje constructivo del conflicto en la escuela .(pp 110-135) 
una tercera persona. Ayuda a las partes a lograr un mayor entendimiento de cómo se perciben los conflictos, las causas subyacentes y entender la visión del otro" (Salcedo e Yves, 2016, p.27), y en su sentido generalmente aceptado, se refiere al establecimiento de un puente comunicacional por parte de un tercero entre partes con tendencias discordantes.

Ahora bien, conforme a la Escuela Internacional de Mediación (2020), las ventajas de este proceso se constriñen a que acarrean menores costes emocionales y económicos, al tiempo que es un procedimiento confidencial y rápido. Puede afirmarse que el alcance de la mediación es que conforme a sus parámetros de aplicación, da pie a la construcción de una comunidad pacífica en tanto que brinda el inicio de la creación de una cultura de respeto, solidaridad y tolerancia, en función de la búsqueda del beneficio para las partes involucradas en una situación que puede caracterizarse como conflictiva. Es por ello que el mediador, al fungir como tercero neutral que asiste en esa negociación que busca la generación de soluciones que provengan de las partes con intereses contrarios, debe cumplir con ciertos requisitos: “...es objetivo y neutral por lo que facilita el diálogo, para que las partes lleguen a una solución satisfactoria para ambas. El mediador no es un árbitro ni un juez, por lo que tampoco juzga ni impone soluciones" (Asociación Europea de Arbitraje, 2019, p.1).

Al asumir todas las consideraciones previas, se llega entonces a consolidar la base para comprender lo que es la mediación educativa o escolar: "la mediación es una forma de resolver conflictos entre dos o más personas, con la ayuda de un tercero imparcial, el mediador. Los mediadores pueden ser los alumnos, profesores, padres”(Sánchez, 2016, p.4) y con ello se vendría a promover un ambiente de armonía en la escuela. Podría decirse entonces que los objetivos de la mediación educativa se adhieren a:

a) Lograr el abordaje constructivo de los elementos tóxicos para las relaciones personales dentro del seno escolar en el área de los conflictos, orientando a la construcción y no a la destrucción, permitiendo transformaciones para, como último fin, acabar con la violencia generada o que potencialmente pudiera suscitarse.

b) Convertir el escenario del conflicto destructivo en una ocasión para encauzar conductas dañinas en el ambiente escolar, conllevándose con ello un aprendizaje aplicable a futuro.

118 Ángel Carmelo Prince Torres: Hacia un modelo de mediación educativa holística para el abordaje constructivo del conflicto en la escuela .(pp 110-135) 
c) Materializar las máximas del currículo por las cuales se pretende la implantación de una cultura democrática.

d) Potenciar destrezas para que los facilitadores trabajen en función de los valores dentro de la institución para su posterior replicación en la comunidad.

e) Propender a la autoevaluación para el crecimiento personal.

También es necesario indicar que una vez reconocido el conflicto, el proceso de mediación escolar podría ceñirse al siguiente protocolo:

Respecto a las personas que están implicadas en el conflicto, es necesario que asuman su parte de responsabilidad en el mismo para poder utilizar esta técnica; es preciso, por tanto, que en ambas partes se dé una voluntad explícita para llevarla a cabo y un compromiso de aceptar el dialogo con la parte enfrentada. En relación a la figura del mediador, se trata de una persona que va a limitar su papel a acompañar a los protagonistas (las personas en conflicto) durante la exploración de la situación, para ayudarles a que averigüen qué necesita cada uno y orientarles en la búsqueda y evaluación de las alternativas de solución. Habitualmente, el mediador suele ser una persona del centro educativo, aunque también se puede acudir a personas externas al mismo. Los requisitos fundamentales que tienen que presentar los eventuales mediadores son los siguientes: por un lado, que se trate de una persona respetada y aceptada por las partes (o «mediados»), por otro, que tenga una buena formación en mediación; adicionalmente, el potencial mediador debe contar con las habilidades pertinentes para facilitar el proceso de mediación, además, naturalmente, de garantizar el cumplimiento estricto de los principios de confidencialidad e imparcialidad en la gestión del proceso (Fundación Créate, 2016, p.1).

Es importante que la persona que funja como mediadora escolar (sea cual sea su rol dentro de la comunidad educativa), asuma su papel con atención a la presencia de indicadores por los cuales se vislumbren actitudes para el cambio, como por ejemplo expresiones parecidas a estas: "a lo mejor podría...", "me complacería si...", "es posible que...", entre otras. Lo expuesto se logra concatenando esta habilidad con la identificación de las secuelas cuando no es fructífera la generación de un acuerdo para que, siempre con tacto, se expliquen las presiones externas que pueden ejercerse si no se arregla 
amistosamente la situación, y que en sí no convienen a las partes. Del mismo modo es importante que se descarten posturas que no vayan de acuerdo con la realidad o que sean extremistas, siempre trayendo a colación información que cuando sea necesario las ayude a recapacitar sobre posiciones precedentes que no resulten alentadoras para consolidar al enfoque ganar-ganar. Todo lo ya mencionado debe realizarlo el coordinador del proceso, teniendo en mente que tiene ante todo que mantener bajo vigilancia las emociones de quienes se mezclan en el hecho acontecido.

Todo este sustrato, se sustentaría de forma general y jurídicamente en el artículo 1 de la Declaración Universal de los Derechos Humanos, donde se apunta que "todos los seres humanos nacen libres e iguales en dignidad y derechos y, dotados como están de razón y de conciencia, deben comportarse fraternalmente los unos con los otros" (Naciones Unidas, 1948, p. 1), lo cual, puede concatenarse con el artículo 26, numeral 2 del mismo texto, en el que se sostiene que,

La educación tendrá por objeto el pleno desarrollo de la personalidad humana y el fortalecimiento del respeto a los derechos humanos y las libertades fundamentales: favorecerá la comprensión, la tolerancia y la amistad entre todas las naciones y todos los grupos étnicos o religiosos; y promoverá el desarrollo de las actividades de las Naciones Unidas para el mantenimiento de la paz (p. 4).

Es así como se observa que con el articulado anterior se promueve la consolidación de una sociedad tolerante y armoniosa, con base en la educación, pues "uno de los más grandes pilares sobre los que ha de fundamentarse la educación contemporánea es, sin lugar a dudas, el de la igualdad" (Lucini, 2000, p.94), y dicha igualdad puede sostenerse en la máxima de justicia de dar a cada quien lo que le corresponde a través de la satisfacción de necesidades, que a su vez traería como consecuencia la cimentación de la paz en las comunidades. Son estas algunas consideraciones a tomar en cuenta para estructurar un modelo de mediación educativa holística.

\section{Aspectos metodológicos}

Este estudio fue realizado bajo el enfoque cualitativo de investigación. Al respecto, se acota que "la investigación bajo el enfoque cualitativo se sustenta en evidencias 
que se orientan más hacia la descripción profunda del fenómeno con la finalidad de comprenderlo y explicarlo" (Sánchez, 2019, p.1), siendo que de esta manera se pretende estudiar a la implementación de la mediación como una forma de reconducir conflictos en la escuela. Por ello, también se circunscribe este texto a un diseño documental en el cual “...el investigador analiza los distintos fenómenos de la realidad obtenidos y registrados por otros investigadores en fuentes documentales" (Brito, 2015, p.8) y en este sentido por lo tanto, se echó mano de la revisión de fuentes secundarias para la consecución de los fines en estas líneas.

Cabe acotar el estudio de documentos se ejecutó con técnicas como la observación, revisión documental, el subrayado la lectura en profundidad, el análisis de textos y la implementación de la lógica para dar coherencia y cohesión a la información recabada. Del mismo modo se hizo uso del análisis crítico, el cual “es la evaluación interna del desarrollo lógico de las ideas, planteamientos o propuestas de un autor. Puede decirse también que es la interpretación personal respecto a la posición de un autor" (Ordóñez, 2015, p.1), por lo que, dentro de este artículo se procedió a concatenar las posiciones en diferentes bibliografías para soportar el trabajo de acuerdo a los requerimientos de quien ha escrito estas líneas.

\section{Resultados: Modelo de mediación educativa holística}

La mediación educativa conduce a la paz dentro del entorno escolar, cuanto más con la problemática actual de la sociedad que se encuentra azotada por la violencia y es precisamente por eso que, en función de construir una comunidad equilibrada y tranquila, debe protegerse al niño o el adolescente pues de esta forma, se asegura la propagación de valores y enseñanzas positivas que así, se perpetuarían en el tiempo. De esta forma, con una visión participativa, democratizadora, epistemológica, axiológica, investigativa, educativa y humanista, la mediación educativa se basa en el holismo, entendido este por la Real Academia Española (2014) de la siguiente manera: "doctrina que propugna la concepción de cada realidad como un todo distinto a las partes que lo componen" (definición 1), al tiempo que se complementa indicando que “...hay

121 Ángel Carmelo Prince Torres: Hacia un modelo de mediación educativa holística para el abordaje constructivo del conflicto en la escuela .(pp 110-135) 
ciertos elementos de espiritualidad en todo lo holístico" (Díaz, 2015,p.1), por lo cual se habría de regir por las siguientes pautas:

a) Debe en primer lugar reconocerse la situación conflictiva, vale decir, identificando a cual tipo de violencia se debe enfrentar, de manera que pueda partir de allí generarse la investigación del trasfondo que impulsó los actos agresivos y puedan abordarse de forma adecuada cuando se presente la comunicación entre las partes y el tercero negociador neutral.

b) Concienciarse sobre la pertinencia de la aplicación del proceso de mediación, es decir, analizar si efectivamente es procedente sólo la aplicación de la mediación.

c) Estudiar las necesidades de los involucrados dentro del espacio académico.

d) Canalizar la expresión de los sentimientos de los involucrados por medio del diálogo.

e) Impulsar la búsqueda de soluciones satisfactorias que provengan desde la propuesta de las mismas partes.

f) Evaluar los logros de la aplicación del proceso de mediación dentro del espacio académico, realizando la retroalimentación que fuera pertinente y observando las modificaciones de la realidad que se han generado para que así, la mediación pueda apreciarse y evolucionar junto con las mutaciones que en la comunidad se presenten como consecuencia de su aplicación.

Por todo lo antes expuesto, al tener como un marco macro a la teoría sobre el conflicto y las generalidades de la mediación, quien escribe estas líneas sostiene que como mediación holística escolar, pueden construirse los siguientes pasos para establecer a esta avenencia en dicho sentido:

a) Impulsar el procedimiento por medio de solicitud de alguno de los involucrados o cualquier agente de la comunidad educativa que haya detectado la situación conflictiva o que prevea la potencialidad del acaecimiento de algún tipo de confrontación entre otros semejantes.

b) Concretar la personificación del mediador, debiendo ser este el docente, el jefe/coordinador docente o alguno de los directivos de la institución (con mérito en la formación pedagógica que se le ha suministrado, aunque también por la abordaje constructivo del conflicto en la escuela .(pp 110-135) 
responsabilidad que tiene dentro de los centros educativos, tomando en cuenta sus destrezas para resolver los conflictos, así como experiencia en el tratamiento de las relaciones interpersonales), ya que, cumpliendo con su elevada misión, son ellos quienes conviven día a día de forma permanente dentro de la escuela junto con otros actores del proceso de aprendizaje.

c) Escoger el lugar dentro del cual se llevaría a cabo la sesión, de preferencia un sitio amplio, bien ventilado e iluminado, dentro del cual pueda conversarse sin barreras comunicacionales físicas y que permita expresar libremente las inquietudes, ideas y deseos sin que existan interrupciones o se pueda filtrar hacia el exterior la información que se está señalando durante la sesión.

d) Una vez escogido el sitio, el mediador, teniendo presente en todo momento que no debe parcializarse con ninguna de las partes y debiendo ante todo fomentar el control de las emociones durante la sesión, abrirá el procedimiento formalmente por medio de un saludo cordial y establecerá las normas rectoras de la sesión, haciendo hincapié con palabras sencillas, en las normas del buen hablante y del buen oyente y es por este motivo que de forma concreta y sin el uso de mayores tecnicismos o vocabulario rebuscado, debe expresarse lo que se desea comunicar a las partes involucradas.

e) Durante la sesión, el mediador debe reforzar en todo momento las cualidades positivas de los participantes, legitimando sus intereses, pero recordándoles sus fortalezas, de manera que se cree un clima de confianza y las partes perciban que son tomadas en cuenta y solamente se persigue su bienestar.

f) Una vez oídas las partes, se retomará su opinión consultando sus deseos y expectativas con respecto a lo que pretenden lograr con la sesión.

g) Posteriormente, se implementaría la técnica de la lluvia de ideas realizada por las partes contrapuestas, de manera que expresen las posibles soluciones que tengan en mente y con la orientación del mediador, se descarten las de más difícil realización.

h) Se realizará y firmará un acuerdo escrito, en donde se pauten los compromisos a los cuales se acordó cumplir, del mismo modo que se asentará el deber de confidencialidad sobre los aspectos desarrollados en la sesión. 
i) Luego se procederá a realizar una retroalimentación final y a recalcar la importancia de mantener relaciones armónicas, la consecuencia de la generación de conductas disruptivas dentro de la institución y la preponderancia de la paz y el amor al prójimo como valores que conllevan al crecimiento personal.

j) Se realizará la despedida cordial.

k) Se recomienda que por tratarse de una escuela y visto que en ella los estudiantes por vía natural son niños y adolescentes, se notifique a los padres y/o representantes de estos, acerca del proceso llevado a cabo, sus implicaciones y beneficios así como la finalidad que se persiguió al realizarla, de manera que puedan contribuir con el éxito de los hechos consumados. La mediación educativa holística, debería llevarse a cabo en tantas sesiones como sea necesario para la consecución de sus fines, en tanto que con la misma, se persigue la orientación de los actores educativos para que comprendan la importancia de la armonía y una convivencia pacífica dentro de la escuela.

El hecho de llevar a cabo el proceso de mediación educativa holística, no implica de algún modo que no haya que procederse echando mano de otras formas correctivas complementarias, como la aplicación de sanciones establecidas en normas jurídicas, puesto que como bien es entendido por las personas la ley no debe ser en ningún caso desacatada, pues la misma regula el comportamiento del hombre en sociedad y hay casos en los que las consecuencias han resultado tan extremas, que requieren de una medida ejemplarizante para que no vuelvan a repetirse. Sin embargo, esto no significa que, en el marco de la mediación, no deba explicarse la razón de esta acción, ya que es necesario que el niño, el adolescente o en definitiva, los implicados, comprendan sus errores para que eviten cometerlos a futuro entrañando con ello un agravamiento de los hechos o incluso la destrucción de su propia persona y de los demás.

En síntesis, el modelo teórico de mediación educativa holística propuesto se atendría primordialmente a los pasos previamente mencionados, integrados con las implicaciones que se han explicado a lo largo de este apartado, resultando de esta forma que se consolide como un medio adecuado de abordaje de controversias. Es pues de este modo como se concreta la aproximación del modelo como una oportunidad para el cambio,

124 Ángel Carmelo Prince Torres: Hacia un modelo de mediación educativa holística para el abordaje constructivo del conflicto en la escuela .(pp 110-135) 
una modificación positiva de la realidad y el inicio para el refuerzo de una comunidad pacífica, que del mismo modo que la mediación en su concepción general debe caracterizarse por ser: a) voluntaria b) confidencial c) célere d) económica (de tiempo y dinero) e) aleccionadora f) participativa y democrática g) sistematizada h) representativa de la autodeterminación de las partes. Por ello, tomando en cuenta los tres modelos fundamentales de la mediación que a continuación se desglosan, pueden proponerse las técnicas que igualmente son implementables en esta negociación holística, que parte de estas concepciones:

a) Modelo tradicional-lineal de Harvard: Establece la satisfacción de necesidades a través de la resolución de situaciones conflictivas, entablando una comunicación lineal en la cual cada parte alternadamente manifiesta una idea y la otra procede a escuchar o no, es decir que "está orientado a la consecución del acuerdo" (Rondón, 2011, p.153). Mientras que se entiende que el conflicto es netamente producto de desacuerdos por lo que considera las necesidades para tratar de generar un consenso apartando a los sujetos de la problemática, fundamentando las resultas en una postura objetiva y sistematizando el curso de los acontecimientos a través de a) un discurso de apertura b) el entendimiento de las perspectivas de los involucrados y sus posturas c) la expresión de los requerimientos d) la producción de alternativas y e) la consolidación de un acuerdo.

b) Modelo Transformativo de Bush y Folger: El cual persigue un acuerdo conjuntamente con el mejoramiento de la ubicación contextual de las personas involucradas realizando una contrastación con el inicio de la situación, pues "este enfoque ve el conflicto como una oportunidad de crecimiento y transformación moral del individuo, plantea que la mediación es la oportunidad perfecta para que los individuos sean más solidarios entre sí” (Isaza, Murgas \& Oñate, 2018, p. 140), modificando las relaciones en conflicto por medio del empoderamiento(entendido como la posibilidad de hacer ver a los sujetos que deben autovalorarse y pueden enfrentar los problemas de su vida, siendo la meta primodial que resulten fortalecidos sin dejar de lado la empatía hacia las situaciones ajenas, replanteando la 
visión de la situación y flexibilizando las posturas para llegar incluso a la generación de una disculpa).

c) Modelo Circular Narrativo de Sara Cobb: Sobre este modelo, se sostiene que "ayuda a resolver creativamente desde una nueva narrativa los conflictos y a la vez, disfrutar con las soluciones elegidas, las cuales son determinadas por las partes en conflicto" (Munuera, 2007, p. 85), por lo cual se afirma que se basa en el fomento de la reflexión a través de la transformación de los acontecimientos de manera que desemboquen en el establecimiento de un acuerdo logrado a través de una comunicación circular entre las partes, en el entendido que no hay un motivo de casualidad exclusivo sino que dicha relación es circular, permitiéndose así que se cree una historia alternativa a la inicial por medio de la aplicación de preguntas circulares estructuradas bajo la óptica de que los hechos no son aislados.

De esta manera, las técnicas susceptibles de ser aplicadas por el mediador o la mediadora en este modelo de mediación educativa holística con el apoyo en el trabajo de Calvo (2012), serían:

a) El parafraseo: Es decir, explicar con palabras propias ideas ya explanadas, lo cual permite la identificación de información relevante, mitigar el recelo entre los involucrados, estar en la seguridad de que lo escuchado es real e igualmente introducir al negociador asistente en el proceso comunicativo.

b) La implementación de sesiones privadas o caucus: que consiste en la reunión del mediador o de la mediadora con cada involucrado o involucrada de forma separada y con paridad de tiempo, de manera que se pueda ahondar en los intereses de las partes, recabar información y del mismo modo entablar la confianza que tan necesaria es para el éxito del proceso.

c) La formulación de preguntas: Teniendo en cuenta que "...las preguntas son las intervenciones básicas fundamentales para la mediación” (Suares, 2018, p. 51), se dice que sirven para determinar los anhelos de las partes. Las preguntas pueden ser de diversos tipos: abiertas (destinadas a obtener respuestas extensas para conocer generalidades y opiniones así como impresiones, como por ejemplo: ¿cómo 
describes el ambiente en tu salón de clases?), cerradas (buscan respuestas concretas, tal como en el caso de la interrogante: ¿quién actuó de este modo?).

También existen las preguntas lineales (son realizadas para dar una orientación al coordinador o la coordinadora de la sesión en cuanto a las propias partes, como en el caso de la pregunta: ¿por qué actuaste así?), circulares (dan pie al desglose de los mismos hechos bajo diferentes representaciones, dilucidando relaciones, hechos, acepciones, emociones y declaraciones de los involucrados, ejemplificándose así: ¿cómo observarías la situación si estuvieras en el lugar del otro?). Por último deben mencionarse las preguntas reflexivas (se fundamentan en el influenciamiento indirecto de las personas en conflicto, realizando las preguntas con hipótesis y dando apertura a nuevos escenarios, siendo un buen ejemplo este: ¿Qué crees que pasaría si..?).

a) Salir al balcón: Se usa cuando se necesita el replanteamiento de la situación conflictiva. Es decir, el mediador se toma un tiempo para establecer mecanismos de redireccionamiento de la sesión, siempre suspendiendo las reacciones.

b) Uso del poder para educar: Cuando el mediador o la mediadora indica a las partes involucradas las consecuencias de sus posturas.

c) La lluvia de ideas: Sobre ella se reseña que consiste en “...utilizar la imaginación y combinar la capacidad creativa del grupo para contar con un abanico de opciones para hacer frente a un problema" (Universia Perú, 2020, p. 1). Así, esta técnica es propuesta por el autor de este artículo, quien considera que para la búsqueda de soluciones bien pudieran los involucrados, una vez desarrollada la sesión, exteriorizar varias ideas y sugerencias de parte y parte, descartando las mismas hasta obtener una solución satisfactoria para ellas.

Con todas estas consideraciones, se establece un canal no sólo de entendimiento entre los seres humanos, sino también de consolidación de una cultura de paz que tan necesaria se hace en la sociedad.

\section{Conclusiones y recomendaciones}

Una vez estructuradas las consideraciones relativas sobre la mediación educativa en este artículo, conviene concluir lo siguiente:

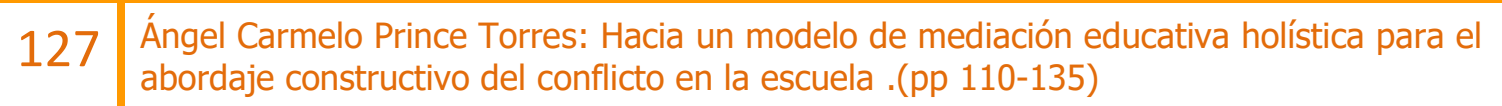


a) La necesidad de estudiar la violencia como un fenómeno socioeducativo latente dentro de las instituciones de educación, se evidencia por la importancia que reviste la interacción en las relaciones interpersonales entre estudiantes. De ninguna manera representa esta la materialización de una labor inútil, sino que por el contrario, es el inicio para la comprensión del trasfondo causal en las conductas agresivas que manifiestan los niños y los adolescentes al enfrentarse por contraposición de sus intereses, siendo esta una realidad que se palpa incluso en aquellas instituciones en las cuales la formación en valores es fundamental para el desempeño de sus actividades diarias.

b) Los conflictos pueden manifestarse de diferentes formas en los ambientes académicos, pero en el proceso de intercambio de ideas y vivencias que se suscita dentro de la escuela, los niños y los adolescente se encuentran en una situación de especial exposición y vulnerabilidad ante el fenómeno, pues se hallan en un proceso de transición y maduración que muchas veces incluso, no les permite discernir entre las conductas erradas y las correctas, por lo que requieren especial atención.

c) La mediación representa un dispositivo eficaz y eficiente por medio del cual, de forma confidencial, con economía de tiempo y respetando los intereses y deseos de las partes en el marco de la orientación de sus planteamientos por parte de un tercero neutral, pueden prevenirse, contenerse o resolverse conflictos que pueden desencadenar la destrucción social. Del mismo modo el establecimiento de un modelo de avenencia desde un punto de vista más holístico, representa un cambio de paradigma pues no sólo se persigue una solución justa al problema planteado, sino también resguardas los sentimientos y anhelos de los involucrados en un evento conflictual.

Así, teniendo en cuenta las percepciones anteriormente desglosadas, puede afirmarse que efectivamente la violencia genera más violencia y por ello, debe desde las instituciones educativas como facilitadoras en la internalización de valores, hacerse frente a esta problemática, siendo la mediación uno de los modos idóneos de abordar el conflicto de forma constructiva, para que de esta manera constituya una oportunidad para cambios positivos en los espacios de convivencia. Es lamentable que la violencia sea una la 
realidad dentro de las instituciones educativas, por lo que la erradicación o prevención de dichos acontecimientos no debe ser concebida como una utopía en el sentido de su imposibilidad de lograrse, sino como una utopía de aquello que es posible para vivir en un mundo mejor.

La violencia es la "desmesura en las relaciones que el ser humano mantiene consigo mismo y con el otro. La violencia reina por doquier" (Cruz, 2000, p. 142) y es por eso que se debe transformar la realidad con disposición a los cambios positivos desde el hogar como primer espacio de socialización, dando lugar a las áreas de reflexión que permitan crear realmente conciencia de que este núcleo es fundamental para la formación de niños y púberes sanos. En segundo lugar, la comunidad debe asimilar la importancia de educación para aplicar vías alternas que permitan dirimir conflictos de forma pacífica, dando pie de este modo a que se siembre una pequeña contribución que, con el tiempo así como con un cambio de actitudes, paradigmas, y concepciones generalizadas es susceptible de replicarse en la conciencia humana, cimentando de esta forma una verdadera noción de hermandad y respeto por los derechos propios y ajenos.

Con todas estas consideraciones en mente, no resulta descabellado recomendar la formación en valores en los propios docentes, e incluso, el estudio en profundidad de sus potencialidades a manera que reciban por medio de charlas, talleres, cursos, el entrenamiento necesario para aplicar las vías alternas de abordaje constructivo del conflicto como medios no jurisdiccionales idóneos para mantener la armonía interpersonal entre los miembros que hacen vida social en la escuela. La mediación no representa la solución absolutista de la problemática que aquí se ha planteado, pero sí es una importante herramienta que se complementa con otros instrumentos cuya génesis se ha materializado para lograr como fin último el bienestar social y la consolidación del amor como principal sentimiento que de forma benéfica, emana del ser humano y que se traduce en riqueza espiritual y tranquilidad incuestionable.

El cambio comienza por el humano mismo y así, es importante que todos los actores sociales estén al tanto de sus derechos, así como sus deberes, comprendiendo que la adhesión a ciertos parámetros de conducta es necesaria para crear una sociedad que construya y no destruya. Sólo resta apuntar lo siguiente a quienes lean estas líneas: sean los 
valientes que luchen, sin importar que en ocasiones parezcan desfallecer para lograr este fin, pues la convicción de un ser humano justo pesa más que la voz vacía de miles injustos.

\section{Referencias}

Asociación Europea de Arbitraje (2019). Mediación. España: Asociación Europea de Arbitraje. Recuperado de https://www.asociacioneuropeadearbitraje.org/mediacion/

Bolaños, D. (2019, septiembre -- octubre). La familia y su influencia en la convivencia escolar. Universidad y Sociedad, (11). dehttp://scielo.sld.cu/scielo.php?script=sci_arttext\&pid=S2218$\underline{36202019000500140 \& \operatorname{lng}=\mathrm{es} \& \mathrm{nrm}=\mathrm{iso} \& \operatorname{lng}=\mathrm{es}}$

Brito, A. (2015). Guía para la elaboración, corrección y asesoramiento de trabajos de investigación. San Tomé: Universidad Nacional Experimental Politécnica de la Fuerza Armada Bolivariana.

Calvo, R. (2012). Del alcance de la mediación. Cataluña: Centre d’ estudisjurídics i formacióespecialitzada.

Couttolenc, J. (2020). Medios alternos de solución de conflictos acortan los juicios. México: Universidad Veracruzana. https://www.uv.mx/prensa/banner/mediosalternos-de-solucion-de-conflictos-acortan-los-juicios/

Cruz, J. (2000). Epítome de Filosofía. Ediciones Colegio Universitario Fermín Toro.

Cuenca, N.(2006). Manual de Mediación Educativa. ¿Cómo formar líderes Democráticos para la Resolución Pacífica de Conflictos? Barquisimeto, Venezuela: Centro de Resolución de Conflictos del Colegio de abogados del Estado Lara.

Díaz, L. (2015). Holística: 5 maneras de cambiar tu vida (a mejor). España: La Razón. https://www.larazon.es/atusalud/objetivo-bienestar/holistica-5-maneras-para-cambiartu-vida-a-mejor-DJ11188478/

Escuela Internacional de Mediación (2020). Ventajas de la mediación ¿las conoces? España: Escuela Internacional de mediación. https://eimediacion.edu.es/sermediador/ventajas-mediacion-conflictos/

Fundación Créate (2016). Los pasos de la mediación escolar. España: Fundación Créate. http://www.fundacioncreate.org/2016/04/07/proceso-la-mediacion-escolar/ 
Galtung, J. (1998). Tras la Violencia 3R: Reconstrucción, Reconciliación, Resolución, Afrontando los Efectos Visibles e Invisibles de la Guerra y la Violencia. Bilbao: Bakeaz, GernikaGogoratuz.

Hicks, D. (2000). Educación para la Paz. Madrid: Ediciones Morata.

Isaza J., Murgas K., y Oñate, M. (2018). Aplicación del modelo transformativo de mediación en la conciliación extrajudicial de Colombia. Revista paz y conflictos (11). DOI:10.30827/REVPAZ.V11I1.6234

Lucini, F. (2000). Temas transversales y educación en valores. Madrid: Grupo Anaya, S.A.

Molina, A. (2020). La importancia de educar para la paz. España: El País. https://elpais.com/elpais/2020/02/02/opinion/1580664370_641392.html. El País.

Munuera, P. (2007). El modelo circular narrativo de Sara Cobb y sus técnicas. PORTULARIA https://eprints.ucm.es/5678/1/_Modelo_circular_narra_P_Munuera.pdf

Naciones Unidas (1948). Declaración Universal de Derechos Humanos (1948).Organización de Naciones Unidas. https://www.un.org/es/universaldeclaration-human-rights/

Olweus, D. (1998). Conductas de Acoso y Amenaza entre Escolares. Ediciones Morata.

Ordóñez, C. (2015). Cómo realizar el análisis crítico de un artículo científico. Guatemala: Universidad San Carlos de Guatemala. https://estudiodental.files.wordpress.com/2015/06/anc3a1lisis-crc3adtico.pdf

Real Academia Española (2014). Diccionario de la Lengua Española (23 ${ }^{\mathrm{a}}$ ed.). España: Real Academia Española. www.rae.es

Rondón, L. (2011). Modelos de Mediación en el mundo Multiétnico. Trabajo Social .13, 153-169 https://revistas.unal.edu.co/index.php/tsocial/article/view/28444/39616

Salcedo A. e Yves, J. (2016). La mediación como herramienta de resolución de conflictos en el sistema educativo dominicano, manual de entrenamiento para facilitadores. Santo Domingo: Ministerio de Educación de República Dominicana.

Sánchez Flores, F.. (2019). Fundamentos epistémicos de la investigación cualitativa y cuantitativa: consensos y disensos. Revista Digital de Investigación en Docencia Universitaria, 13(1), 102-122. https://dx.doi.org/10.19083/ridu.2019.644 
Sánchez, I. (2016). El conflicto y la mediación en la comunidad educativa. España: Universidad de La Rioja https://publicaciones.unirioja.es/catalogo/online/CIFETS_2016/Monografia/pdf/TC39 6.pdf.

Suares, M. (2018). Procesos de mediaciones y técnicas. Colombia: Pontificia Universidad Javeriana Cali. http://proyectos.javerianacali.edu.co/cursos_virtuales/posgrado/maestria_asesoria_fa miliar/mediacion/modulo3/unidad3/Suares\%20(2018)Procesos\%20de\%20Mediaci+\% C2\%A6n\%20y\%20T+\%C2\%AEcnicas\%20Cap\%205.pdf

Unicef (2018). La mitad de los adolescentes del mundo sufre violencia en la escuela. UNICEF. $\quad$ https://www.unicef.org/es/comunicados-prensa/la-mitad-de-losadolescentes-del-mundo-sufre-violencia-en-la-escuela

Universia Perú (2020). Cómo hacer una lluvia de ideas exitosa. Perú: Universia Perú. https://noticias.universia.edu.pe/educacion/noticia/2015/03/18/1121714/5-consejosrealizar-lluvia-ideas-clase.html

Universidad Internacional de la Rioja (2020). ¿Cuáles son los tipos de violencia escolar? España: UNIR. https://www.unir.net/educacion/revista/noticias/violenciaescolar/549204985723/

Universidad Internacional de Valencia (2018). ¿Por qué se producen los conflictos en el aula? España: Universidad Internacional de Valencia. https://www.universidadviu.com/por-que-se-producen-los-conflictos-en-el-aula/.

Weil, P. (1996). Introducción al Pensamiento Complejo. Gedis 